\title{
LEPROT and LEPROTL1 cooperatively decrease hepatic growth hormone action in mice
}

\author{
Thierry Touvier, ${ }^{1,2,3,4}$ Françoise Conte-Auriol, 5 Olivier Briand,1,2,3,4 Céline Cudejko,1,2,3,4 \\ Réjane Paumelle,1,2,3,4 Sandrine Caron,1,2,3,4 Eric Baugé,,1,2,3,4 Yves Rouillé, 1,4,6 \\ Jean-Pierre Salles, ${ }^{5}$ Bart Staels, ${ }^{1,2,3,4}$ and Bernard Bailleul',2,3,4
}

${ }^{1}$ Université Lille Nord de France, Lille, France. ${ }^{2}$ INSERM U545, Lille, France. ${ }^{3}$ UDSL, Lille, France. ${ }^{4}$ Institut Pasteur de Lille, Lille, France. ${ }^{5}$ INSERM U563, Université Paul Sabatier, Hôpital des Enfants CHU de Toulouse, Toulouse, France. ${ }^{6}$ CNRS-UMR8161, Institut de Biologie de Lille, Lille, France.

\begin{abstract}
Growth hormone (GH) is a major metabolic regulator that functions by stimulating lipolysis, preventing protein catabolism, and decreasing insulin-dependent glucose disposal. Modulation of hepatic sensitivity to GH and the downstream effects on the GH/IGF1 axis are important events in the regulation of metabolism in response to variations in food availability. For example, during periods of reduced nutrient availability, the liver becomes resistant to GH actions. However, the mechanisms controlling hepatic GH resistance are currently unknown. Here, we investigated the role of 2 tetraspanning membrane proteins, leptin receptor overlapping transcript (LEPROT; also known as OB-RGRP) and LEPROT-like 1 (LEPROTL1), in controlling GH sensitivity. Transgenic mice expressing either human LEPROT or human LEPROTL1 displayed growth retardation, reduced plasma IGF1 levels, and impaired hepatic sensitivity to GH, as measured by STAT5 phosphorylation and Socs 2 mRNA expression. These phenotypes were accentuated in transgenic mice expressing both proteins. Moreover, gene silencing of either endogenous Leprot or Leprotl1 in H4IIE hepatocytes increased GH signaling and enhanced cell-surface GH receptor. Importantly, we found that both LEPROT and LEPROTL1 expression were regulated in the mouse liver by physiologic and pathologic changes in glucose homeostasis. Together, these data provide evidence that LEPROT and LEPROTL1 influence liver GH signaling and that regulation of the genes encoding these proteins may constitute a molecular link between nutritional signals and $\mathrm{GH}$ actions on body growth and metabolism.
\end{abstract}

\section{Introduction}

Growth hormone $(\mathrm{GH})$ is the main regulator of postnatal growth. Indeed, patients with GH deficiency or GH receptor (GHR) mutations (Laron syndrome) exhibit severe postnatal growth retardation. GH also exerts metabolic actions on muscle and adipose tissue: it stimulates peripheral lipolysis, displays protein-sparing actions, and decreases insulin-dependent glucose disposal (1). These effects contribute to spare protein and glucose stores at the expense of increased fat utilization. During periods of reduced nutrient availability, the liver becomes resistant to $\mathrm{GH}(2,3)$. As a consequence, hepatic GH-induced IGF1 secretion decreases, which in turn increases pituitary GH secretion because of lack of feedback inhibition. This open-loop feedback system, defined as the GH/IGF1 axis, may constitute an advantageous mechanism providing an optimal protection of protein and carbohydrate stores critical to survival under conditions of energy shortage.

The mechanism of hepatic GH resistance has been extensively studied. In rodent liver, impairment of GH signaling and reduced cell-membrane $G b r$ abundance have been described during fasting $(2,3)$ and streptozotocin-induced (STZ-induced) diabetes $(4,5)$. Refeeding and insulin therapy in these models restore hepatocyte Gbr abundance (3, 4). In HuH7 hepatoma cells, insulin increases GH signaling and cell-surface Gbr abundance (6). Low circulating levels of high-affinity GH-binding protein (GHBP) in malnourished individuals and type 1 diabetes mellitus (T1DM) patients

Conflict of interest: The authors have declared that no conflict of interest exists. Citation for this article: J. Clin. Invest. 119:3830-3838 (2009). doi:10.1172/JCI34997. suggest a similar mechanism of liver GH resistance in humans (7). Indeed, the level of plasma GHBP, which derives mainly from proteolytic cleavage and shedding of liver GHR, is believed to be a reflection of hepatic GHR expression levels. However, the molecular partners regulating membrane GHR abundance following changes in nutrient availability have not been identified.

The GHR belongs to the superfamily of cytokine receptors and, like other membrane receptors, homodimerizes in a ligand-independent manner (8). GHR signal transduction is mainly mediated by tyrosine kinase activation of JAK2 (9). Subsequently, the receptor and several signaling proteins are phosphorylated on key tyrosine residues, resulting in the activation of several signaling pathways. The STAT5 is a key mediator in GH-stimulated gene activation implicated in somatic growth (9) and is involved in GH lipolytic action (10). GHR cell-surface abundance and the magnitude of the initial GH-induced signaling are controlled by internalization and lysosomal degradation of the GHR/JAK2 complex (11). Later on, intracellular STAT activation is repressed by diverse processes including the GH-induced expression of SOCS (12). After internalization, the GHR ends up in the endosomal-lysosomal system, where it is degraded (11).

The leptin receptor overlapping transcript (LEPROT, initially named $O B-R G R P)(13)$ belongs to a family of genes that includes 1 other member in higher cells, LEPROT-like 1 (LEPROTL1) (14), and 1 member in Saccharomyces cerevisiae, vacuolar protein sorting 55 (VPS55) (15). The LEPROT family encodes small proteins of 131-140 amino acids with 4 potential transmembrane domains. The importance of the tetraspanning membrane proteins Vps55p 
A
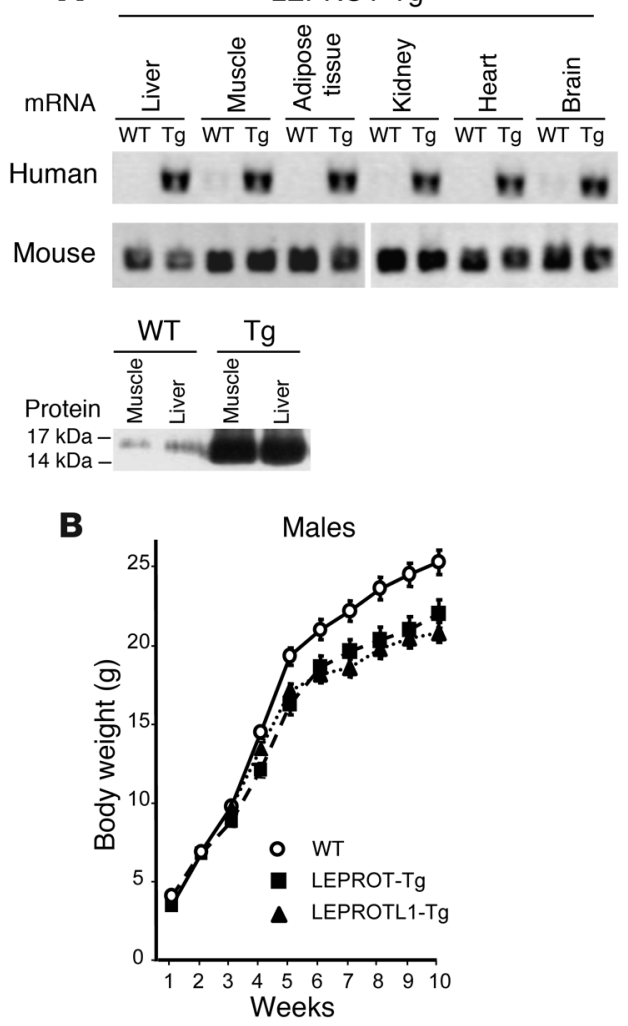

LEPROTL1-Tg
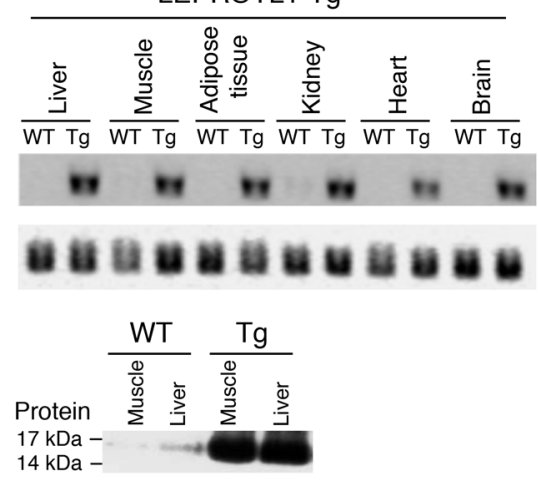

Females

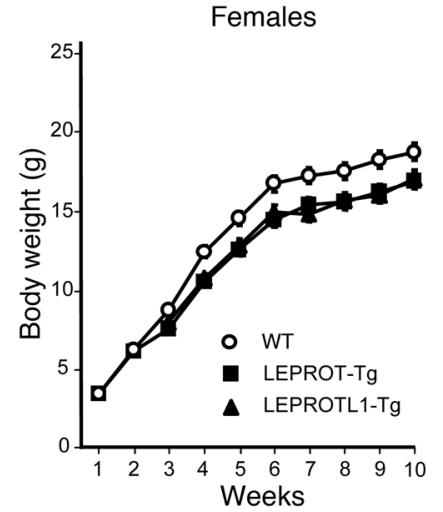

Figure 1

Transgenic expression of $\angle E P R O T$ and LEPROTL1 induces growth retardation. (A) Top: human transgenic and mouse endogenous $L E P R O T$ and $L E P R O T L 1$ mRNA were detected by RT-PCR in the indicated tissues of WT and transgenic (left, LEPROTTg; right, LEPROTL1-Tg) mice. The mouse Leprot mRNA samples were run on the same gel but were noncontiguous. Bottom: LEPROT and LEPROTL1 proteins were analyzed by Western blot in muscle and liver of WT and transgenic (Tg: left, LEPROT; right, LEPROTL1) mice. (B) Growth curves of WT (circles), LEPROTTg (squares), and LEPROTL1-Tg (triangles) male and female mice. Data shown are mean \pm SEM. and LEPROT in protein trafficking to the vacuole/lysosome of eukaryotic cells has been highlighted by the phenotype of yeast with a disrupted VPS55 gene (vps55 $\triangle$ strain) (15). Indeed, the vps $55 \Delta$ strain displays normal endocytosis of the endocytic marker uracil permease, but a drastic delay of its vacuolar degradation. In addition, human LEPROT (hLEPROT) expression in yeast results in a localization pattern similar to that of Vps55p, being mainly present in late endosomes, and corrects the vacuolar targeting defects of carboxypeptidase $\mathrm{Y}(\mathrm{CPY})$ in the vps55 $\Delta$ cells (15). These data suggest a phylogenetically conserved role and, accordingly, suggest that LEPROT may be involved in the downregulation of membrane protein levels and their targeting from late endosomes to lysosomes (15). It is likely that LEPROT family members are part of functional protein complexes that include at least 1 small membrane protein with 4 putative transmembrane domains. Indeed, $\mathrm{Vps} 55 \mathrm{p}$ interacts with $\mathrm{Vps} 68 \mathrm{p}$, a small tetraspan protein (16). Recently, it has been demonstrated in vitro that LEPROT negatively regulates leptin receptor cell-surface expression and that in vivo LEPROT silencing in mouse hypothalamic arcuate nucleus increases leptin signaling (17). In mammals, LEPROT and LEPROTL1 mRNA are widely expressed, including in metabolic tissues such as muscle and liver $(13,14,18)$.

In this study, using transgenic mice, we show that both LEPROT and LEPROTL1 induce growth retardation and decrease GH responsiveness in liver and isolated hepatocytes. This effect is not restricted to liver since expression of these genes also reduces GH sensitivity in myoblast cells. Furthermore, we show that hLEPROT and hLEPROTL1 decrease cell-surface Gbr expression. Importantly, LEPROT and LEPROTL1 display (a) a cooperative effect in decreasing hepatic GH sensitivity and (b) an increased expression in liver under (patho)physiological conditions of GH resistance, as in T1DM. These data indicate that LEPROT and LEPROTL1 play a role in the control of hepatic GH resistance and are molecular links between nutritional signals and GH action.

\section{Results}

Growth retardation in transgenic mice overexpressing hLEPROT and hLEPROTL1. To identify the function of the LEPROT and LEPROTL1 proteins, transgenic mice strains (LEPROT-Tg and LEPROTL1-Tg) were generated overexpressing the $L L E P R O T$ or LLEPROTL1 genes driven by a ubiquitous promoter in the C57BL/6 genetic background. Transgenic LEPROT and LEPROTL1 expression was evidenced by RNA analysis in several tissues, which also express these genes endogenously (Figure 1A). Western blot analysis using antibodies against the $\mathrm{C}$ termini sequences of LEPROT or LEPROTL1, which are conserved between the human and mouse orthologs, revealed a $16-\mathrm{kDa}$ band, which was more intense in muscle and liver from transgenic than from WT mice (Figure 1A).

The LEPROT and LEPROTL1 transgenic pups developed normally, and adult male and female mice were fertile and apparently healthy. Morphometric parameters were measured at birth and followed up to 10 weeks of age (Figure 1B and Table 1). At birth, no difference in body weight was observed between transgenic and WT littermate mice. In contrast, at 3 weeks of age, both the male and female LEPROT-Tg and LEPROTL1-Tg mice had a lower body weight than their WT littermates, which was statistically significant at 5 weeks of age $(P<0.05)$. At 10 weeks of age, female and male transgenic mice displayed an approximately $10 \%$ lower body weight compared with WT mice. Liver weight was similarly reduced in 10-week-old LEPROT-Tg and LEPROTL1-Tg mice (Table 1). 
Table 1

Transgenic expression of LEPROT and LEPROTL1 reduces body weight and plasma IGF1 levels

\begin{tabular}{lcccccc} 
& \multicolumn{3}{c}{ Males } & \multicolumn{3}{c}{ Females } \\
& WT & LEPROT & LEPROTL1 & WT & LEPROT & LEPROTL1 \\
Body weight $(\mathrm{g})$ & $24.6 \pm 1.1$ & $22.2 \pm 1.2^{\mathrm{A}}$ & $21.9 \pm 1.1^{\mathrm{B}}$ & $20.5 \pm 0.9$ & $18.1 \pm 0.8^{\mathrm{A}}$ & $18.2 \pm 0.7^{\mathrm{B}}$ \\
Body length $(\mathrm{cm})$ & $9.5 \pm 0.1$ & $9.1 \pm 0.1^{\mathrm{C}}$ & $9.0 \pm 0.1^{\mathrm{C}}$ & $8.8 \pm 0.1$ & $8.3 \pm 0.1^{\mathrm{C}}$ & $8.4 \pm 0.1^{\mathrm{C}}$ \\
Liver weight $(\mathrm{g})$ & $1.20 \pm 0.08$ & $1.02 \pm 0.07^{\mathrm{C}}$ & $1.07 \pm 0.06^{\mathrm{C}}$ & $0.86 \pm 0.06$ & $0.82 \pm 0.05^{\mathrm{C}}$ & $0.81 \pm 0.04^{\mathrm{C}}$ \\
AT weight $(\mathrm{g})$ & $0.56 \pm 0.06$ & $0.60 \pm 0.09$ & $0.53 \pm 0.08$ & $0.38 \pm 0.07$ & $0.39 \pm 0.07$ & $0.36 \pm 0.05$ \\
IGF1 $(\mathrm{ng} / \mathrm{ml})$ & $374 \pm 15$ & $323 \pm 19 \mathrm{C}$ & $303 \pm 22^{\mathrm{C}}$ & $289 \pm 16$ & $228 \pm 15^{\mathrm{C}}$ & $234 \pm 20^{\mathrm{C}}$
\end{tabular}

Body, liver, and adipose tissue (AT; corresponds to both inguinal and perigonadal adipose tissues) weight; noseanus length; and plasma IGF1 levels of WT, LEPROT-Tg, and LEPROTL1-Tg male and female mice at 10 weeks of age $(n=10-12)$. Data shown are mean \pm SEM. ${ }^{A} P<0.01 ;{ }^{B} P<0.001 ;{ }^{C} P<0.05$ versus WT; Wilcoxon's $t$ test.

compared with that of WT mouse hepatocytes (-27\% and $-32 \%$ respectively; Figure 2B), whereas Ghr mRNA levels were not modified in these conditions (Figure 2C).

These data show that LEPROT-Tg and LEPROTL1-Tg mice display an impairment of hepatic GH-induced STAT5 phosphorylation associated with decreased GH-binding capacity, whereas $G h r$ gene expression is unchanged.

LEPROT and LEPROTL1

However, the ratio of liver/body weight was similar to that in WT mice. The body length (nose to anus) of 10-week-old LEPROT-Tg and LEPROTL1-Tg mice was significantly shorter than that of WT mice. Interestingly, plasma levels of IGF1, a marker of hepatic GH action, were significantly reduced in transgenic mice (LEPROT-Tg and LEPROTL1-Tg mice, males: $-14 \%$ and $-19 \%$; females: $-21 \%$ and $-19 \%$ ) compared with WT littermate mice (Table 1).

Thus, overexpression of hLEPROT and hLEPROTL1 results in general growth retardation of both male and female mice, which is associated with decreased IGF1 plasma levels, which suggests that these proteins influence the GH/IGF1 axis.

GH-induced STAT5 phosphorylation and GH binding are lower in livers of LEPROT-Tg and LEPROTL1-Tg mice. To determine whether LEPROT and LEPROTL1 influence GH signaling in the liver, we measured tyrosine phosphorylation of STAT5 (p-STAT5) in LEPROT$\mathrm{Tg}$ and LEPROTL1-Tg livers after intravenous injection of GH. p-STAT5 was not detected in control livers after injection of a saline solution. The GH-induced P-STAT5/STAT5 ratio was reduced by $34 \%$ and $38 \%$ in livers from LEPROT-Tg and LEPROTL1$\mathrm{Tg}$ mice, respectively, compared with WT mice (Figure 2A). To explore whether LEPROT and LEPROTL1 may affect cell-surface Ghr abundance, [ $\left.{ }^{125} \mathrm{I}\right] \mathrm{GH}$-binding assays were performed on primary hepatocytes isolated from WT, LEPROT-Tg, and LEPROTL1$\mathrm{Tg}$ mice incubated in the presence of high concentrations of human prolactin to saturate the lactogenic sites. The GH-binding capacity of LEPROT-Tg and LEPROTL1-Tg hepatocytes was markedly reduced decrease GH responsiveness. To determine whether LEPROT or LEPROTL1 interferes directly with GH signaling in hepatocytes, GHstimulated p-STAT5 was studied in mouse primary hepatocytes infected with adenoviruses expressing LEPROT, LEPROTL1, or control GFP and subsequently incubated with or without GH (Figure 3A). Treatment of GFP adenovirus-infected primary hepatocytes with $\mathrm{GH}$ resulted in a strong increase of p-STAT5 compared with untreated hepatocytes (Figure 3A). Infection with the LEPROT- or LEPROTL1-expressing adenoviruses did not influence the total STAT5 content, whereas GH-induced STAT5 phosphorylation was significantly lower (p-STAT5/STAT5 LEPROT: $0.2 \pm 0.06$; LEPROTL1: $0.5 \pm 0.1$ ), when compared with GFP adenovirus-infected cells (Figure 3A). In addition, induction of Socs 2 expression after GH treatment was significantly attenuated in LEPROT- and LEPROTL1-infected cells compared with GFP-infected primary hepatocytes (3.8-, 1.5-, and 2.1-fold increase for GFP, LEPROT, and LEPROTL1, respectively; Figure 3B). Similar results were obtained in the nonhepatic, mouse myoblast C2C12 cell line (5.4-, 2.5-, and 3.1-fold increase for GFP, LEPROT, and LEPROTL1, respectively; Figure 3C).

To determine whether Leprot or Leprotl1 knockdown provokes the opposite effect, gene-silencing experiments were performed using Leprot and Leprotl1 siRNA in rat H4IIE hepatocytes. 72 hours after transfection, Leprot or Leprotl1 siRNA-transfected cells exhibited 60\% lower Leprot or Leprotl1 mRNA, respectively, than control siRNA-transfected cells (data not shown). Under

\section{Figure 2}

LEPROT and LEPROTL1 decrease GH responsiveness and cell-surface $\mathrm{GH}$ binding in liver. (A) Liver phospho-STAT5/ STAT5 ratio after intravenous $\mathrm{GH}$ or saline (PBS) injection ( $n=4$ mice per group), (B) specific $h \mathrm{GH}$ binding in primary hepatocytes at $4^{\circ} \mathrm{C}$ ( $n=3$ mice per group), and (C) liver endogenous mouse Ghr mRNA expression ( $n=3$ mice per group) in WT and LEPROTTg and LEPROTL1-Tg mice. ${ }^{*} P<0.05$ versus WT; Wilcoxon's $t$ test. Data shown are mean $\pm S D$.
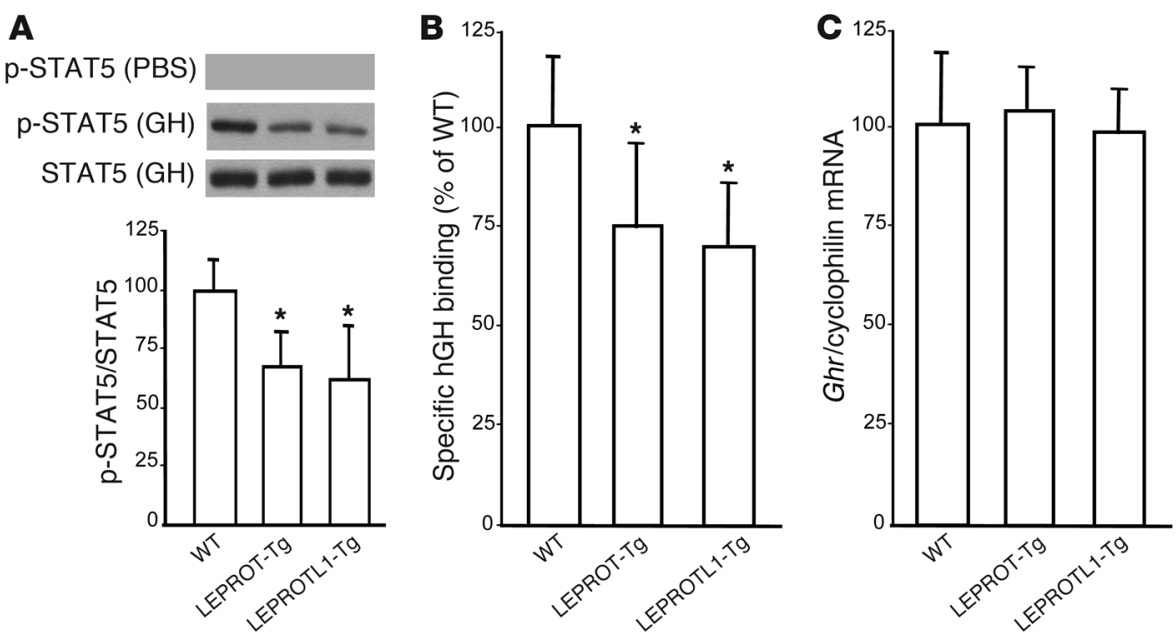
A
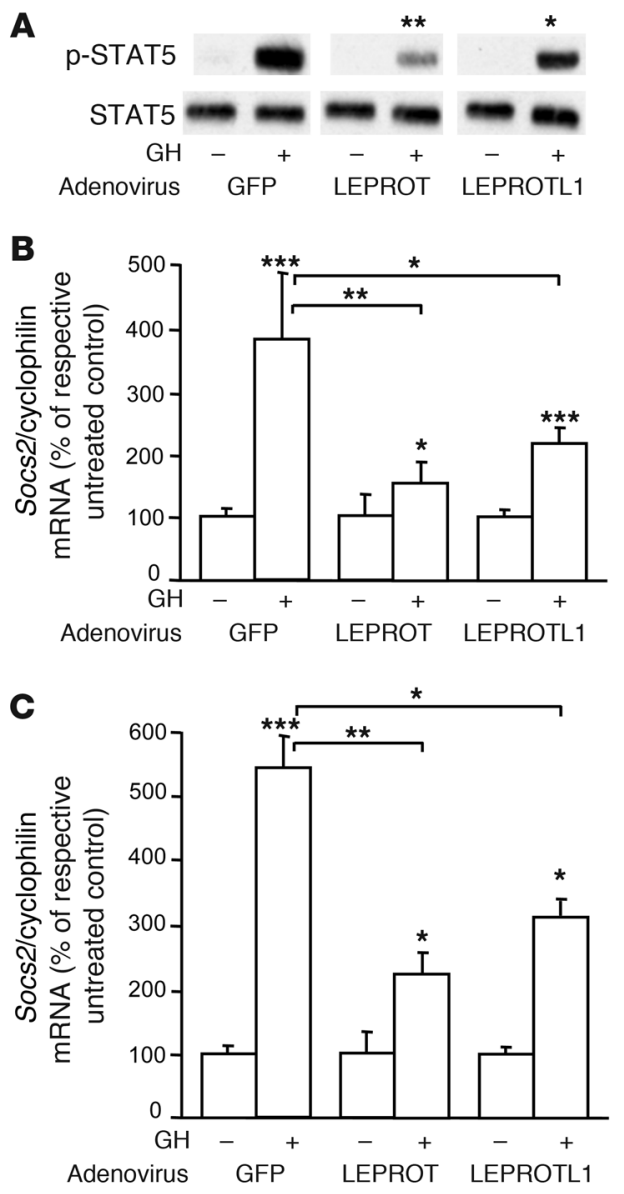

these conditions of silencing, higher specific GH binding (about 2 -fold higher than in control siRNA-transfected cells; Figure 4A) was observed. GH treatment resulted in increases of p-JAK2 levels (Figure 4B), p-STAT5 levels (Figure 4C), and Socs2 mRNA expression (respectively, 2.1- and 1.4-fold higher than in control siRNA-

\section{Figure 3}

LEPROT and LEPROTL1 decrease GH signaling. Mouse primary hepatocytes (A and $\mathbf{B}$ ) and mouse $\mathrm{C} 2 \mathrm{C} 12$ myoblast cells $(\mathbf{C})$ were infected with GFP-, LEPROT-, or LEPROTL1-expressing adenoviruses and subsequently incubated with (+) or without (-) GH. Basal and GHinduced p-STAT5/STAT5 ratio $(\mathbf{A})$ and Socs2 mRNA (B and $\mathbf{C}$ ) were measured by Western blot and Q-PCR, respectively. The blot is representative of 3 independent experiments; lanes were run on the same gel but were noncontiguous. Quantifications were performed from 3 independent experiments. ${ }^{* *} P<0.001 ;{ }^{* *} P<0.01$; ${ }^{*} P<0.05$ versus respective control, Wilcoxon's $t$ test. Data shown are mean \pm SD.

transfected cells; Figure 4D) in Leprot and Leprotl1 siRNA-transfected cells compared with control siRNA-transfected cells. These data demonstrate that both LEPROT family members directly decrease GH signaling and expression of GH target genes.

Overexpression of LEPROT and LEPROTL1 downregulates cell-surface GHR abundance but does not enhance soluble GHBP. To determine whether LEPROT and LEPROTL1 act by modulating GHR expression, COS-7 cells were transfected with human GHR (hGHR) and hLEPROT or hLEPROTL1 expression vectors. As assessed by realtime quantitative PCR (Q-PCR), LEPROTs did not modify transcriptional expression of the hGHR-expressing vector (data not shown). Assessment of cell-surface GHR abundance by performing a cell-surface-specific $\left[{ }^{125} \mathrm{I}\right] \mathrm{GH}$-binding assay at $4^{\circ} \mathrm{C}$ showed that both LEPROT and LEPROTL1 expression reduced GH binding by $75 \%$ and $70 \%$, respectively (Figure $5 \mathrm{~A}$ ). Using increasing amounts of LEPROT or LEPROTL1 vectors, a dose-dependent effect was observed (data not shown). Scatchard analysis was performed to determine the Bmax, which evaluates maximum GHbinding potential, a Kd-independent value. A decreased Bmax was observed in LEPROT- and LEPROTL1-transfected cells (0.02 and $0.01 \mathrm{nM}$, respectively) compared with control-transfected cells $(0.4 \mathrm{nM})$, indicating that cell-surface GHR expression is reduced. As a control, cell-surface-specific EGF-binding capacity was not altered in similar experiments using EGFR-transfected cells (Figure 5B), indicating that LEPROT and LEPROTL1 act on GHR in a specific manner. To determine whether LEPROT and

\section{Figure 4}

Leprot and Leprotl1 silencing increase GH signaling. H4IIE hepatic cells were transfected with control, Leprot, or Leprotl1 siRNAs. (A) Specific hGH binding at room temperature ( $n=3$ per group). (B) p-JAK2, JAK2 (C), p-STAT5, STAT5, and (D) Socs2 mRNA expression in control, LEPROT, or LEPROTL1 siRNA-transfected cells after incubation with (+) or without (-) GH were measured by Western blot and Q-PCR, respectively. The lanes on panel $\mathbf{C}$ were run on different gels. ${ }^{* * *} P<0.001$; ${ }^{\star} P<0.05$ versus respective treated control, Wilcoxon's $t$ test $(n=3)$. Data shown are mean \pm SD.

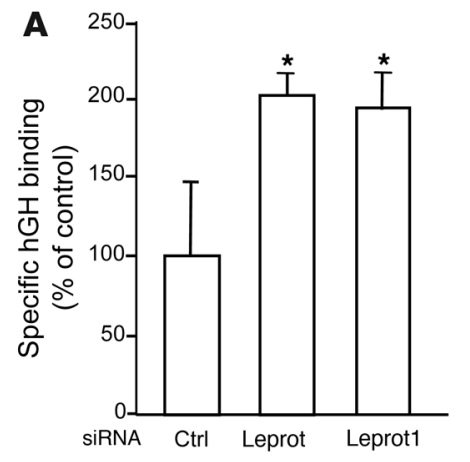

B
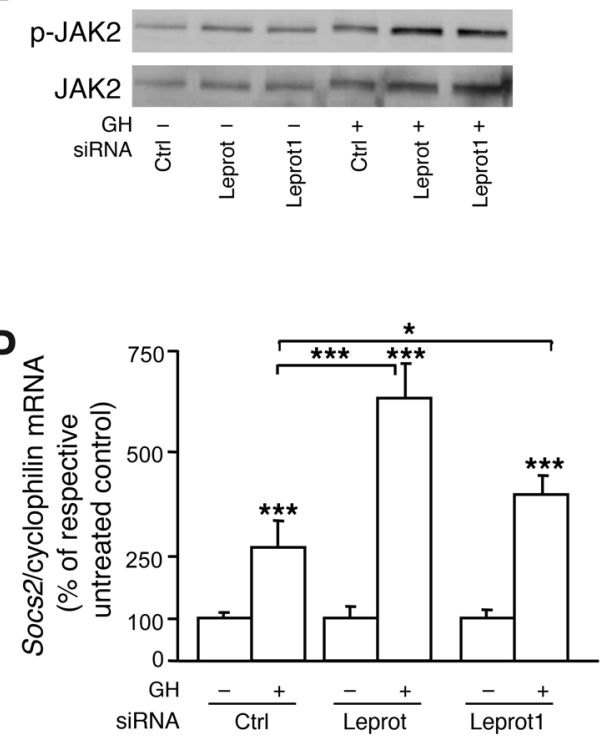

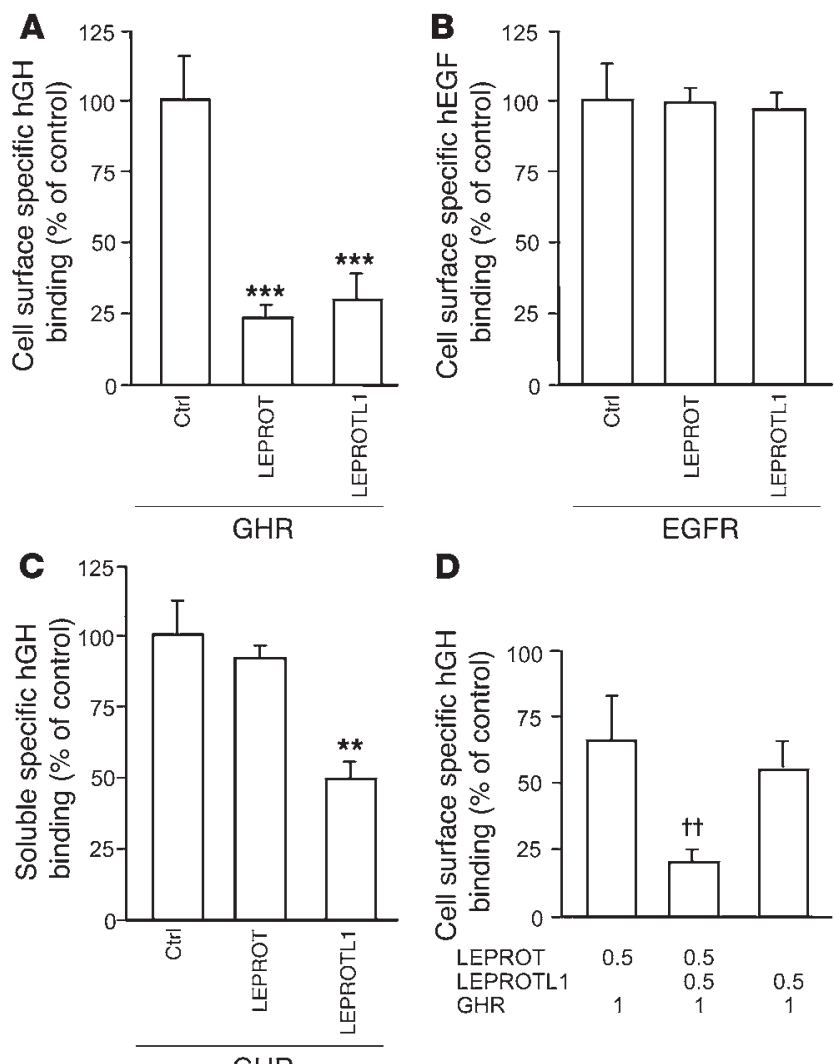

D

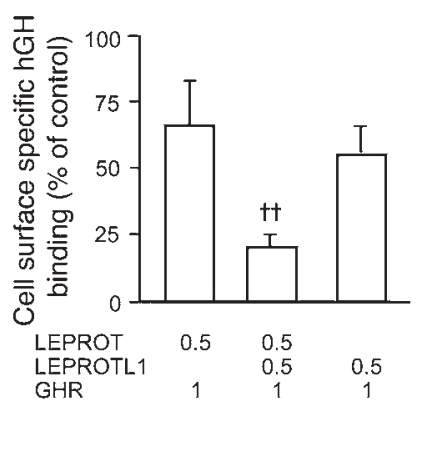

LEPROTL1 affect GHR proteolysis at the cell surface, soluble GHBPs were measured by analyzing $\left[{ }^{125} \mathrm{I}\right] \mathrm{GH}$ binding in transfected-cell medium. LEPROTL1 decreased by $50 \%$ soluble GHBP abundance, whereas LEPROT had no effect (Figure 5C). Finally, cotransfection of both LEPROT and LEPROTL1 significantly

\section{Figure 5}

LEPROT and LEPROTL1 decrease cell-surface GH binding but not $\mathrm{hEGF}$ binding without increasing soluble $\mathrm{GH}$ binding formation. COS-7 cells were cotransfected with GHR (A, C, and D) or EGFR (B). Specific $\mathrm{hGH}$ binding (A and D) and hEGF binding (B) at $4^{\circ} \mathrm{C}$ confined to the cell surface were determined in the presence of LEPROT or LEPROTL1 expression vectors $(1.25 \mu \mathrm{g})$ compared with control vectors ( $\mathbf{A}$ and $\mathbf{B}$ ) or both LEPROT and LEPROTL1 expression vectors (0.5 $\mu \mathrm{g}$ each) compared with single transfection of each LEPROT or LEPROTL1 (0.5 $\mu \mathrm{g}$; D). Specific hGH binding corresponding to soluble GHR in medium from A was determined (C). ${ }^{* \star} P<0.001 ;{ }^{\star \star} P<0.01$ versus control transfected cells; ${ }^{\dagger} P<0.01$ versus single transfected cells, Wilcoxon's $t$ test $(n=3)$. Data shown are mean \pm SD.

reduced cell-surface $\left[{ }^{125} \mathrm{I}\right] \mathrm{GH}$ binding compared with single LEPROT or LEPROTL1 transfection (Figure 5D).

The studies in COS cells suggest that both members of the LEPROT family specifically decrease plasma-membrane GHR without influencing GHR gene expression and without increasing the release of soluble GHR in medium.

Hepatic Leprot and Leprotl1 mRNA expression is increased by fasting and STZ-induced diabetes and decreased by insulin. Next, it was determined whether liver LEPROT and LEPROTL1 expression is controlled by (patho)physiological conditions associated with hepatic GH insensitivity and decreased GHR cell-surface abundance, i.e., fasting and T1DM. In WT mice, liver LEPROT and LEPROTL1 mRNA expression levels increased after 1 day of fasting (Figure $6 \mathrm{~A})$. Moreover, 12-hour post-refeeding with a high-carbohydrate diet decreased hepatic expression of both LEPROT and LEPROTL1 (Figure 6A). Plasma insulin levels were significantly different in ad libitum, fasting, and 12-hour refed mice $(1.21 \pm 0.26,0.33 \pm 0.18$, $6.93 \pm 3.82 \mathrm{ng} / \mathrm{ml}$, respectively). Western blots were performed on protein extracts from liver of WT mice fed ad libitum or after fasting. Using both antibodies against the $\mathrm{C}$ termini of LEPROT and LEPROTL1, which display cross-reactivity, together, a significant

\section{Figure 6}

Regulation of Leprot and Leprot/1 mRNA expression by nutritional status, diabetes status, and insulin treatment. (A) Endogenous Leprot and Leprotl1 mRNA levels in livers of WT female mice fed ad libitum, subjected to 24 hours fasting, and refed for 12 or 24 hours with a highcarbohydrate diet ( $n=8$ mice per group) were measured by Q-PCR. (B) Endogenous Leprot and Leprotl1 protein levels in livers of WT female mice fed ad libitum or subjected to a 24 hour fasting were measured by Western blot, using antibodies against the C-ter of both Leprot and Leprotl1 $(n=3)$. (C) Endogenous Leprot and Leprot/1 mRNA levels were measured in livers from control or STZ-induced T1DM mice (STZ; glycemia $>400 \mathrm{mg} / \mathrm{dl}$ ) ( $n=8$ mice per group). (D) Endogenous Leprot and Leprotl1 mRNA levels were measured in H4IIE hepatocytes treated for 24 hours with insulin $(10 \mathrm{nM})$ by Q-PCR. ${ }^{* *} P<0.01 ;{ }^{\star} P<0.05$ versus control, Wilcoxon's $t$ test $(n=5)$. Data shown are mean \pm SEM.
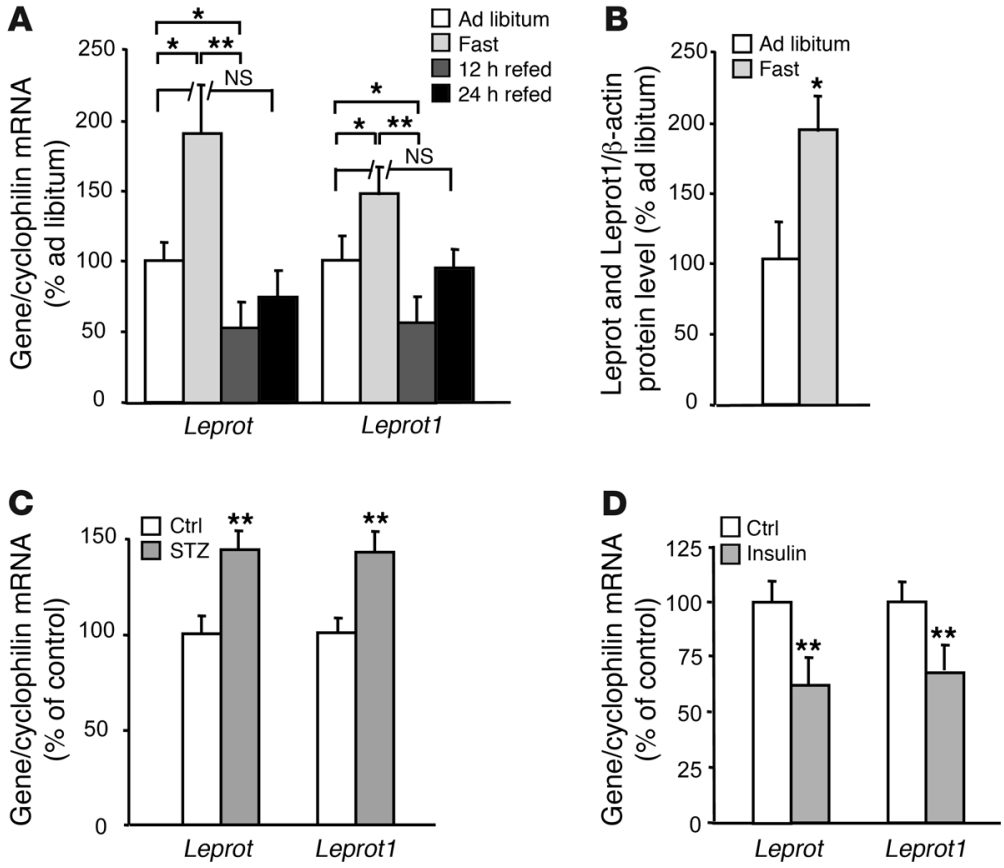
A

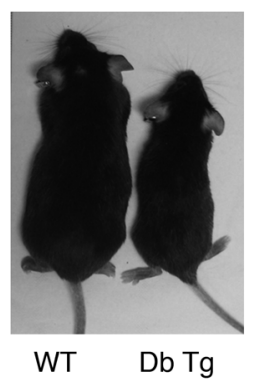

C

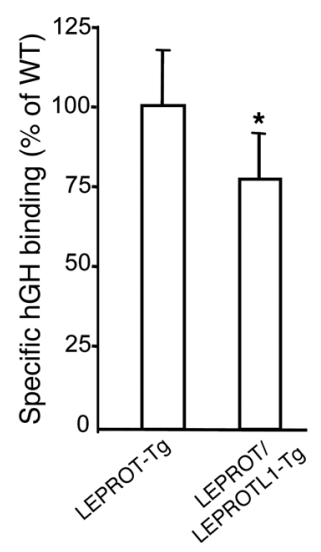

B

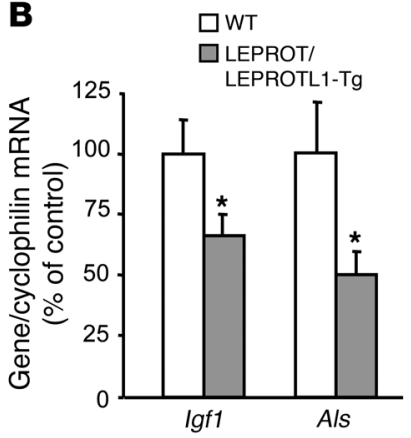

D

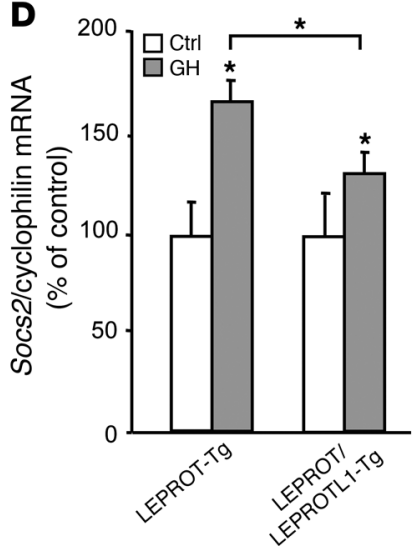

increased expression of total Leprot and Leprotl1 was observed in livers of 24-hour-fasted compared with ad libitum-fed mice (Figure 6B). STZ-induced diabetic mice displayed increased liver Leprot and Leprotl1 mRNA expression levels (Figure 6C) and a $35 \%$ decrease of plasma IGF-I levels compared with control mice (control, $362 \pm 15 \mathrm{ng} / \mathrm{ml}$; STZ, $238 \pm 48 \mathrm{ng} / \mathrm{ml} ; P<0.05$ ). Finally, insulin treatment significantly reduced Leprot and Leprotl1 mRNA levels in H4IIE cells (Figure 6D). These data show that a simultaneous increase in the liver expression of both genes occurs in (patho)physiological conditions associated with liver GH resistance and that insulin may participate in this regulation.

Cooperative effects of LEPROT and LEPROTL1 in transgenic mice. Finally, to investigate the effect of simultaneous overexpression of both genes, double-transgenic mice expressing both LEPROT and LEPROTL1 (LEPROT/LEPROTL1-Tg) were generated. These double-transgenic male mice (LEPROT/LEPROTL1-Tg) displayed significantly lower body weight, body size, and plasma IGF1 levels compared with WT mice (Figure 7A and Table 2). The LEPROT/LEPROTL1-Tg phenotype was more pronounced compared with that of single-transgenic mice. Compared with WT mice, body weight and plasma IGF1 levels of LEPROT/LEPROTL1-Tg mice were approximately $39 \%$ and $34 \%$ lower, respectively, compared with $10 \%-14 \%$ in either LEPROT-Tg or LEPROTL1-Tg mice (Tables 1 and 2). No differences in daily food intake/body weight ratio, GH, and insulin plasma levels were observed between LEPROT/LEPROTL1-Tg and WT littermate mice. In addition, hepatic mRNA expression of GH-regulated genes, such as acid-labile subunit $(A l s)$ and Igf1, was significantly decreased in livers of LEPROT/ LEPROTL1-Tg (Figure 7B) compared with those of WT mice. This effect was not observed in either LEPROT-Tg or LEPROTL1-Tg mice (data not shown). To further support the exacerbated pheno-

\section{Figure 7}

LEPROT/LEPROTL1 double-transgenic mice display an exacerbated growth defect phenotype and liver $\mathrm{GH}$ resistance. (A) Picture of 10-week-old male WT and LEPROT/LEPROTL1 Tg mice. (B) Liver Igf1 and Als mRNA expression levels after 6 hours of fasting in WT $(n=6)$ and LEPROT/LEPROTL1-Tg mice $(n=4)$. (C) Specific cell-surface hGH binding ( $n=3$ mice per group) and (D) Socs2 mRNA levels (with or without $\mathrm{GH}$ ) in primary hepatocytes isolated from LEPROT-Tg and LEPROT/LEPROTL1-Tg mice. ${ }^{*} P<0.05$, LEPROT/LEPROTL1Tg versus WT (B) or versus LEPROT-Tg (C and D), Wilcoxon's $t$ test. Data shown are mean \pm SD.

type of the double-transgenic mice versus single-transgenic mice, specific GH binding and GH-induced Socs 2 mRNA expression were compared in hepatocytes derived from LEPROT-Tg and LEPROT/ LEPROTL1-Tg. Compared with LEPROT alone, the expression of LEPROT and LEPROTL1 resulted in decreased cell-surface GH binding and GH-induced Socs 2 expression (Figure 7, C and D).

These data show that LEPROT and LEPROTL1, without influencing food intake and plasma GH levels, display cooperative effects on body growth, plasma IGF1 levels, surface GH binding, and hepatic GH-regulated genes.

\section{Discussion}

Using a transgenic approach, we identify a role for the 2 members of the LEPROT gene family as negative regulators of GHinduced STAT5 signaling leading to decreased body growth associated with decreased plasma IGF1 levels and Ghr cell-surface abundance. Our results on primary and H4IIE hepatocytes provide evidence that LEPROT and LEPROTL1 are directly involved at the cellular level in the modulation of GH-induced STAT5 signaling. The role of hepatic GHR and STAT5 signaling in the control of plasma IGF1 levels is well documented. Indeed, a decrease of plasma IGF1 I has been reported in 10-week-old Ghr-deficient mice (19) and in mice with a liver Ghr-signaling impairment by partial or hepatic-specific deletion of the Stat5aStat5b locus $(20,21)$. Human patients with GHR mutations and homozygous STAT5b mutations display markedly reduced serum IGF1 concentrations (22). However, the role of hepatic GH sensitivity and liver IGF1 expression in postweaning body growth remains controversial. Indeed, whereas liver-specific

\section{Table 2}

Transgenic expression of both LEPROT and LEPROTL1 display an exacerbated growth defect phenotype and decreased plasma IGF1 levels

\begin{tabular}{lcc}
\hline & WT & LEPROT/LEPROTL1-Tg \\
Body weight $(\mathrm{g})$ & $24.6 \pm 1.0$ & $14.9 \pm 0.9^{\mathrm{A}}$ \\
Body length $(\mathrm{cm})$ & $9.60 \pm 0.20$ & $8.75 \pm 0.05 \mathrm{~B}$ \\
IGF1 $(\mathrm{ng} / \mathrm{ml})$ & $365 \pm 19$ & $242 \pm 38^{\mathrm{C}}$ \\
$\mathrm{GH}(\mathrm{ng} / \mathrm{ml})$ & $22 \pm 5$ & $21 \pm 3$ \\
$\mathrm{Fl} / \mathrm{BW}(\mathrm{g} / \mathrm{day} / \mathrm{g})$ & $0.22 \pm 0.01$ & $0.23 \pm 0.01$ \\
Insulin $(\mathrm{ng} / \mathrm{ml})$ & $1.1 \pm 0.7$ & $1.0 \pm 0.4$
\end{tabular}

Body weight, nose-anus length, IGF1, GH, and insulin plasma levels and the daily food intake/body weight ratio (FI/BW) in WT and male transgenic LEPROT/LEPROTL1 mice at 10 weeks of age $(n=5$ mice by group). Data shown are mean \pm SEM. ${ }^{A} P<0.001 ;{ }^{B} P<0.01 ;{ }^{C} P<0.05$ versus WT; Wilcoxon's $t$ test. 
deletion of Ghr has no effect on total body growth (23), (a) liverspecific overexpression of IGF1 enhances somatic growth and partially prevents the effects of GH deficiency (24) and (b) disruption of 2 liver GH target genes by crossing Als knockout and liver Igf1-deficient mice results in growth-retarded mice (25). In these transgenic models, the increased pituitary GH secretion due to the lack of IGF1 feedback action may contribute to some extent to the control of body growth. However, it has been shown that GH signaling in extrahepatic tissues also participates in the control of body growth, as demonstrated in mice with musclespecific deletion of the STAT5a-STAT5b locus (26). Together, the decreased GH signaling in liver, the lowered plasma IGF1, the unchanged GH in LEPROTs-Tg mice, the reduced hepatic expression levels of Als and Igf1 in double LEPROT/LEPROTL1$\mathrm{Tg}$ mice, and the regulation of GH signaling in myoblast cells by LEPROTs are in agreement with the proposal that ubiquitous expression of LEPROT and LEPROTL1 in the transgenic mice induces growth retardation, in part as a consequence of a decrease of GH-induced STAT5 signaling. However, it cannot be excluded that LEPROTs also modify events other than GHinduced STAT5 signaling that could modify body growth. In particular, in addition to the STAT5 pathway, GH-induced JAK2 phosphorylation results in the activation of the PI3K and MAPK pathways. Collectively, these pathways mediate the growth-promoting and metabolic actions of GH. The latter signaling pathway is altered in $50 \%$ of the short-stature children with Noonan syndrome (27). Further experiments are required to properly address whether LEPROTs act on such additional pathways.

Our results show that both cell-surface GHR abundance and GH-induced STAT5 signaling are decreased by both LEPROT and LEPROTL1. Until now, no function was described for LEPROTL1. A similar negative regulation on leptin receptor cell-surface expression and leptin-induced STAT3 signaling by LEPROT expression has been described (17). However, there is no evidence that GH-induced STAT signaling is only a direct consequence of cell-surface receptor abundance. Indeed, if we consider that (a) heterozygous Ghr-deficient mice (+/-) have normal plasma IGF1 levels despite a $50 \%$ decrease of Ghr mRNA expression and total $\mathrm{GH}$ binding in liver (19) and that (b) GH-induced STAT5 signaling lasts intracellularly after GHR endocytosis (28), then the duration of intracellular signaling may also be of crucial importance. For some receptors, endocytic organelles, as intracellular stations, may participate in signaling specificity and regulation (29). It is noteworthy that endocytosis and subsequent lysosomal degradation of GHR seem to be the main determinants of GHR availability (30) and inhibitors of lysosomal function markedly reduce GHR degradation and prolong the half-life of both GHR and its ligands $(11,31)$. Both the GHR and the leptin receptor are degraded by the lysosome $(11,32)$. Thus, it is possible that LEPROTs decreased GH signaling by shortening the half-life of the intracellular endocytosed GHRs and the duration of intracellular GH-mediated signaling as well as reducing cell-surface GHR abundance by decreasing or inhibiting its recycling. In this respect, we showed that decreased GHR cell-surface abundance occurs without changes in GHR mRNA expression and with no increased soluble GHR formation. This is in line with the current knowledge of the cellular localization and the function of Vps55p and LEPROTs. In yeast, Vps55p and hLEPROT are localized in the late endosome and participate in the vacuolar targeting of CPY (15). Vps55p also participates in the downregulation of the pheromone receptor ste3p (16). It has been suggested that LEPROT plays a role in late endocytotic steps (17). And finally, both LEPROTs are localized in endosomes (Y. Rouillé, unpublished observations).

Whereas hLEPROT and hLEPROTL1 display 67\% sequence identity, it may be proposed that they are not functionally redundant and they may act at a different and distinct step on the GHR functional activity. This is suggested by the cooperative effect of these proteins on body growth and plasma IGF1. In contrast, generation of LEPROT or LEPROTL1 homozygous transgenic mice did not worsen the growth defect phenotype (data not shown). In addition, LEPROT or LEPROTL1 expression has a different effect on soluble GHR generation. Recently, it has been shown that Vps68p interacts with Vps55p, the yeast homolog of LEPROT, to mediate a step in the endosomal maturation process (16). Ste3p downregulation was similarly delayed in both vps68 and vps55 mutants. Because both Vps55p and Vps68p are represented by 2 homologs in human and mouse, it has been proposed that these 4 proteins in higher cells may form distinct functionally divergent complexes (16). In addition, there is evidence that these proteins may be subject to posttranslational modifications. Indeed, Vps55p is phosphorylated and interacts with ser/thr kinases, (33) and conserved ser/thr residues exist in the intracellular loops and $\mathrm{C}$ and $\mathrm{N}$ termini of all LEPROT family members. The existence of both partners and posttranslational modifications, which are not modified in the transgenic models, and also the postendocytic localization of LEPROT and LEPROTL1 may explain the relatively moderate change in GH sensitivity and growth phenotype with single gene expression and also the cooperative effect of LEPROT and LEPROTL1.

Our results show that LEPROT and LEPROTL1 cooperatively decrease liver GH sensitivity and that the expression of both genes is increased in livers of fasted and STZ-induced diabetic mice. In mice, and probably also in humans, liver GH resistance induced by prolonged fasting and T1DM is characterized by a decrease of both cell-surface GHR abundance and GH signaling (2-4). Similar features are observed in LEPROT transgenic mice. Thus, it is tempting to speculate that the increased expression of both LEPROT and LEPROTL1 may participate in liver GH resistance under conditions of fasting and T1DM. The fact that plasma IGF1 levels, the main marker of hepatic GH resistance, are lowered to a similar extent in the double-transgenic mice and the STZ-induced T1DM WT mice $(242 \pm 38 \mathrm{ng} / \mathrm{ml}$ versus $238 \pm 48$ $\mathrm{ng} / \mathrm{ml}$ ) supports this hypothesis. In addition, we show that insulin in H4IIE hepatocytes decreases LEPROT and LEPROTL1 expression. Consequently, it is likely that insulin participates in the modulation of LEPROT and LEPROTL1 expression in livers of mice during fasting, during refeeding a high-carbohydrate diet, and in T1DM mice with STZ-induced destruction of pancreatic $\beta$ cells, conditions that result in altered insulin plasma levels. High concentrations of insulin inhibit GH-induced STAT5 signaling in H4IIE hepatocytes (34), whereas low concentrations of insulin increase $\mathrm{GH}$-induced signaling and cell-surface $\mathrm{GH}$ binding (6). Moreover, insulin has a positive effect on GH sensitivity in vivo, probably by maintaining liver GHR levels $(4,35)$. In T1DM patients and STZ-treated rodents, insulin deficiency is correlated with hepatic $\mathrm{GH}$ resistance, which is associated with reduced levels of circulating GHBP in patients and decreased liver cell-surface Ghr in rodents (36). In STZ-treated rats, circulating IGF1 is reduced as is GH-binding capacity, whereas insu- 
lin treatment restores IGF1 levels and GH binding $(4,37)$. The hepatic regulation of both LEPROT and LEPROTL1 by insulin may hence participate in the (patho)physiological GH resistance under conditions of fasting and T1DM.

A mechanism that controls tissue GH sensitivity and body growth under conditions of food restriction provides a selective advantage to limit the final body-weight expansion and long-term energy expenditure. Dynamic modulation of hepatic GH sensitivity provides important means to cope with situations of food deprivation. The identification of 2 membrane tetraspanning proteins inhibiting GH sensitivity and their regulation by nutritional status provides a considerable novelty in our understanding of the mechanism of GH sensitivity control.

\section{Methods}

LEPROT and LEPROTL1 transgenic mice. All experiments were performed with the approval of the Institut Pasteur de Lille review board. Transgenes were composed of the open reading frame of hLEPROT or hLEPROTL1 flanked by the cytomegalovirus enhancer, the chicken $\beta$-actin promoter sequence, and the rabbit $\beta$-globin polyadenylation sequence. All experiments on transgenic animals were performed with 1 LEPROT-Tg strain and 2 independent LEPROTL1-Tg strains in the C57BL/6J background. Crossing LEPROT-Tg and LEPROTL1-Tg strains generated double-transgenic mice overexpressing LEPROT and LEPROTL1 (LEPROT/LEPROTL1-Tg).

Cohorts of mice were weighed at weekly intervals from birth to 10 weeks of age. IGF1 and GH levels were determined in serum from orbital bleeds using an ELISA kit (ImmunoDiagnostic systems and Diagnostic Systems Laboratories, respectively), and plasma glucose concentrations were determined using Glucotrend 2 (Roche Diagnostics) on tail bleeds after a 6-hour fast.

Intravenous GH injections were performed in mice anesthetized with pentobarbital. In brief, the abdominal cavity was opened, and vehicle or recombinant human GH (hGH) (MAXOMAT; Sanofi) was injected at a dose of $0.5 \mathrm{mg} / \mathrm{kg}$ of body weight in the vena cava. 10 minutes later, mice were sacrificed, and livers were removed and stored at $-80^{\circ} \mathrm{C}$ for subsequent analysis.

WT mice were submitted to a 24-hour fast followed by refeeding with a high-carbohydrate diet as previously described (38). Diabetic mice (STZ; glycemia $>400 \mathrm{mg} / \mathrm{dl}$ ) were selected 3 weeks after a daily intraperitoneal injection of STZ (Sigma-Aldrich) ( $40 \mathrm{mg} / \mathrm{kg}$ of body weight). Control mice received an injection of the solvent ( $0.05 \mathrm{M}$ sodium citrate).

$D N A$ and RNA extraction and qualitative PCR and Q-PCR analysis. Total RNA was isolated from organs or cells using the TRIzoL reagent according to the manufacturer's instructions (Invitrogen). RNA reverse transcription and PCR amplifications were performed as described (39). Primers used in these studies are indicated here: LEPROT-Tg and LEPROTL1-Tg (GGCCTTATTCGTCCTGA and CAAATACTGCCCCCTCTTGTTCTATT, respectively, with TATCCGTCGGACGTGGACT); Leprot (GGGCTGACTTTTCTTATGCTG, CCCAGTGGTGAAGAAATACGC); Leprotl1 (GCCCTTCCGATATACAACCA, CTCCTTACACGCGTTGC); Socs2 (CTGCCTGGAAGTCACAGGGTA, TGGAGAAAAAGAACCAGCCG); GHR (CCCAGGTGAGCGACATTAC, GGCAGAGTGAGACCATTTCC); Ghr (CGATTCACCAAGTGTCGTTC, TCAGGGCATTCTTTCCATTC); Igf1 (AGCGGGCTGCTTTTGTAGG, TTACTTCAACAAGCCCACAGG); Als (GCGCTCTTGTGTGGCTTGG, GCTGTGAtGAGGTTGCGGTC); and cyclophilin (CATACGGGTCCTGGCATCTTGTCC, TGGTGATCTTCTTGCTGGTCTTGC).

Protein extraction and Western blot analysis. Polyclonal antisera were obtained by immunizing rabbits with a C-terminal dodecapeptide of LEP-
ROT or LEPROTL1. P-STAT5 (Tyr694) antibody was purchased from Cell Signaling Technology, and STAT5 and JAK2 antibody was purchased from Santa Cruz Biotechnology Inc. P-JAK2 was purchased from Invitrogen's BioSource division. Protein lysates were separated either by $18 \%$ SDS-PAGE for LEPROT and LEPROTL1 or 10\% SDS-PAGE for other protein analysis, transferred onto PVDF membrane (Hybond-P; GE Healthcare), probed with specific antibodies at 1:1,000 overnight at $4^{\circ} \mathrm{C}$, and revealed using the horseradish peroxidase-conjugated anti-rabbit or anti-goat secondary antibody 1:10,000 from Chemicon. They were then developed with an enhanced chemiluminescence Western blot detection kit (Amersham ECL plus Western blotting Detection System; GE Healthcare).

Mouse primary hepatocyte isolation, cell culture, and adenoviral experiments. Mouse primary hepatocytes were isolated by collagenase perfusion from the livers of mice as previously described (39). Cos-7, H4IIE, and C2C12 cell lines were grown on petri dishes in DMEM supplemented with $10 \%$ FBS, gentamicin $(50 \mu \mathrm{g} / \mathrm{ml})$ at $37^{\circ} \mathrm{C}$ in an atmosphere of $5 \% \mathrm{CO}_{2}$. Recombinant defective adenovirus vectors expressing LEPROT, LEPROTL1, or GFP were generated by homologous recombination in Escherichia coli as described (40) and amplified in HER-911 cells (41). For adenoviral experiments, hepatocytes from WT mice were seeded at a density of $0.75 \times 10^{6}$ cells/well in collagen-coated 6 -well plates (BD Biosciences). After 1 day, adenoviral infections were performed at an MOI of 3 and 20 for hepatocytes and C2C12, respectively, for 6 hours. 24 hours later, cells were treated with $500 \mathrm{ng} / \mathrm{ml} \mathrm{hGH}$ (Maxomat; Sanofi) for 10 minutes (proteins) or 3 hours (RNA).

RNA interference. siGENOME SMARTpool siRNA rat Leprot (L-09587801) and Leprotl1 (L-088926-01) were purchased from Dharmacon. Rat hepatoma H4IIE cells were transfected using the transfection reagent DharmaFECT Reagent 4 (T-2004-02). 72 hours after transfection in serum-free medium, cells were treated with $500 \mathrm{ng} / \mathrm{ml} \mathrm{hGH}$ for $10 \mathrm{~min}$ utes or 3 hours to determine JAK2 and STAT5 phosphorylation and Socs2 expression respectively.

Binding studies. COS-7 cells were transfected with hGHR and hLEPROT or hLEPROTL1 expression vectors using FuGene reagent (Roche) according to the manufacturer's instructions. $\left.{ }^{125} \mathrm{I}\right] \mathrm{GH}$ labeling was as described previously using the chloramine $\mathrm{T}$ method, and analysis of GH binding to the cell surface was performed as described (42). GH binding to surface, used as an estimate of total cellular content of functional GHRs, was performed as already described (6). Detection and quantification of the soluble forms of GHR were performed on centrated medium with [ $\left.{ }^{125} \mathrm{I}\right] \mathrm{GH}$ incubation and gel analysis as already described (42). For GH-binding studies in hepatocytes, $10^{6}$ cells were incubated with $10^{5} \mathrm{cpm}$ human $\left[{ }^{125} \mathrm{I}\right] \mathrm{GH}$ in the presence of $2 \mu \mathrm{g} / \mathrm{ml}$ prolactin to limit lactogenic binding. The incubation mixture was placed at $20^{\circ} \mathrm{C}$ for 120 minutes under continuous rotary shaking. The incubation was stopped by washing the cells with ice-cold PBS. The cells were lysed in $0.1 \mathrm{~N} \mathrm{NaOH}$ for radioactivity measurement. Specific binding of $\left[{ }^{125} \mathrm{I}\right] \mathrm{GH}$ or $\left[{ }^{125} \mathrm{I}\right] \mathrm{EGF}$ was calculated as the difference between total and nonspecific binding (in the presence of $2 \mu \mathrm{g} / \mathrm{ml}$ of nonradiolabeled recombinant hGH or hEGF).

Statistics. Statistical significance of variations among the different experimental groups was determined by Wilcoxon's $t$ test (JMP, SAS Institute). $P$ values less than 0.05 were considered significant.

\section{Acknowledgments}

We thank the staff of the Experimental Genetic Laboratory of Institut Pasteur de Lille and the SEAT (Service d'Expérimentation Animale et de Transgenèse, UPS44 CNRS, Villejuif) for collaboration in the generation of the LEPROT-Tg and LEPROTL1-Tg mice, respectively. The mouse mutant lines LEPROT-Tg and LEPROTL1-Tg, named Tg (CMV_ACTB/hLEPROT) and Tg (CMV_ 
ACTB/hLEPROTL1), respectively, are deposited in the European Mouse Mutant Archive (EMMA) and available under EM:02245 and EM:02246. Archiving was funded by the EC FP7 Capacities Specific Program. We acknowledge Pfizer for logistical support of the Centre de Recherche sur la Croissance de Toulouse, Toulouse, France. This work was supported by grants from the Région NordPas de Calais/FEDER.

1. LeRoith, D., and Yakar, S. 2007. Mechanisms of disease: metabolic effects of growth hormone and insulin-like growth factor 1. Nat. Clin. Pract. Endocrinol. Metab. 3:302-310.

2. Beauloye, V., et al. 2002. Impairment of liver $\mathrm{GH}$ receptor signaling by fasting. Endocrinology. 143:792-800.

3. Postel-Vinay, M.C., Cohen-Tanugi, E., and Charrier, J. 1982. Growth hormone receptors in rat liver membranes: effects of fasting and refeeding, and correlation with plasma somatomedin activity. Mol. Cell. Endocrinol. 28:657-669.

4. Baxter, R.C., Bryson, J.M., and Turtle, J.R. 1980 Somatogenic receptors of rat liver: regulation by insulin. Endocrinology. 107:1176-1181.

5. Chen, N.Y., et al. 1995. Effects of streptozotocin treatment in growth hormone (GH) and GH antagonist transgenic mice. Endocrinology. 136:660-667.

6. Leung, K.C., Doyle, N., Ballesteros, M., Waters, M.J., and Ho, K.K. 2000. Insulin regulation of human hepatic growth hormone receptors: divergent effects on biosynthesis and surface translocation. J. Clin. Endocrinol. Metab. 85:4712-4720.

7. Baumann, G. 2001. Growth hormone binding protein 2001. J. Pediatr. Endocrinol. Metab. 14:355-375.

8. Gent, J., van Kerkhof, P., Roza, M., Bu, G., and Strous, G.J. 2002. Ligand-independent growth hormone receptor dimerization occurs in the endoplasmic reticulum and is required for ubiquitin system-dependent endocytosis. Proc. Natl. Acad. Sci. U. S. A. 99:9858-9863.

9. Lanning, N.J., and Carter-Su, C. 2006. Recent advances in growth hormone signaling. Rev. Endocr. Metab. Disord. 7:225-235.

10. Fain, J.N., Ihle, J.H., and Bahouth, S.W. 1999. Stimulation of lipolysis but not of leptin release by growth hormone is abolished in adipose tissue from Stat5a and b knockout mice. Biochem. Biophys. Res. Commun. 263:201-205.

11. van Kerkhof, P., and Strous, G.J. 2001. The ubiquitin-proteasome pathway regulates lysosomal degradation of the growth hormone receptor and its ligand. Biochem. Soc. Trans. 29:488-493.

12. Davey, H.W., McLachlan, M.J., Wilkins, R.J., Hilton, D.J., and Adams, T.E. 1999. STAT5b mediates the GH-induced expression of SOCS-2 and SOCS-3 mRNA in the liver. Mol. Cell. Endocrinol. 158:111-116.

13. Bailleul, B., Akerblom, I., and Strosberg, A.D. 1997. The leptin receptor promoter controls expression of a second distinct protein. Nucleic Acids Res. 25:2752-2758.

14. Huang, Y., et al. 2001. Cloning and characterization of a novel human leptin receptor overlapping transcript-like 1 gene (LEPROTL1). Biochim. Biophys.

Received for publication January 11, 2008, and accepted in revised form September 23, 2009.

Address correspondence to: Bernard Bailleul, INSERM U545, Institut Pasteur de Lille, 1 rue du Professeur Calmette, BP 245, Lille 59019, France. Phone: 33-3-20-87-71-25; Fax: 33-3-20-87-7198; E-mail: Bernard.Bailleul@pasteur-lille.fr.

Acta. 1517:327-331

15. Belgareh-Touze, N., Avaro, S., Rouille, Y., Hoflack, B., and Haguenauer-Tsapis, R. 2002. Yeast Vps55p, a functional homolog of human obesity receptor gene-related protein, is involved in late endosome to vacuole trafficking. Mol. Biol. Cell. 13:1694-1708.

16. Schluter, C., et al. 2008. Global analysis of yeast endosomal transport identifies the Vps55/68 sorting complex. Mol. Biol. Cell. 19:1282-1294.

17. Couturier, C., et al. 2007. Silencing of OB-RGRP in mouse hypothalamic arcuate nucleus increases leptin receptor signaling and prevents diet-induced obesity. Proc. Natl. Acad. Sci. U. S. A. 104:19476-19481.

18. Mercer, J.G., et al. 2000. B219/OB-R 5'-UTR and leptin receptor gene-related protein gene expression in mouse brain and placenta: tissue-specific leptin receptor promoter activity. J. Neuroendocrinol. 12:649-655.

19. Zhou, Y., et al. 1997. A mammalian model for Laron syndrome produced by targeted disruption of the mouse growth hormone receptor/binding protein gene (the Laron mouse). Proc. Natl. Acad. Sci.U. S. A. 94:13215-13220.

20. Teglund, S., et al. 1998. Stat5a and Stat5b proteins have essential and nonessential, or redundant, roles in cytokine responses. Cell. 93:841-850.

21. Holloway, M.G., et al. 2007. Loss of sexually dimorphic liver gene expression upon hepatocyte-specific deletion of Stat5a-Stat5b locus. Endocrinology. 148:1977-1986.

22. Kofoed, E.M., et al. 2003. Growth hormone insensitivity associated with a STAT5b mutation. N. Engl. J. Med. 349:1139-1147.

23. Fan, Y., et al. 2009. Liver-specific deletion of the growth hormone receptor reveals essential role of growth hormone signaling in hepatic lipid metabolism. J. Biol. Chem. 284:19937-19944.

24. Liao, L., et al. 2006. Liver-specific overexpression of the insulin-like growth factor-I enhances somatic growth and partially prevents the effects of growth hormone deficiency. Endocrinology. 147:3877-3888.

25. Yakar, S., et al. 2002. Circulating levels of IGF-1 directly regulate bone growth and density. J. Clin. Invest. 110:771-781.

26. Klover, P., and Hennighausen, L. 2007. Postnatal body growth is dependent on the transcription factors signal transducers and activators of transcription $5 \mathrm{a} / \mathrm{b}$ in muscle: a role for autocrine/paracrine insulin-like growth factor I. Endocrinology. 148:1489-1497.

27. Binder, G. 2009. Noonan syndrome, the Ras-MAPK signalling pathway and short stature. Horm. Res. 71(Suppl. 2):64-70.

28. Alves dos Santos, C.M., van Kerkhof, P., and Strous, G.J. 2001. The signal transduction of the growth hormone receptor is regulated by the ubiquitin/ proteasome system and continues after endocytosis. J. Biol. Chem. 276:10839-10846.

29. Miaczynska, M., Pelkmans, L., and Zerial, M. 2004. Not just a sink: endosomes in control of signal transduction. Curr. Opin. Cell Biol. 16:400-406.

30. Strous, G.J., and van Kerkhof, P. 2002. The ubiquitin-proteasome pathway and the regulation of growth hormone receptor availability. Mol. Cell. Endocrinol. 197:143-151.

31. van Kerkhof, P., Smeets, M., and Strous, G.J. 2002. The ubiquitin-proteasome pathway regulates the availability of the GH receptor. Endocrinology. 143:1243-1252.

32. Belouzard, S., Delcroix, D., and Rouille, Y. 2004. Low levels of expression of leptin receptor at the cell surface result from constitutive endocytosis and intracellular retention in the biosynthetic pathway. J. Biol. Chem. 279:28499-28508.

33. Ptacek, J., et al. 2005. Global analysis of protein phosphorylation in yeast. Nature. 438:679-684.

34. Ji, S., Guan, R., Frank, S.J., and Messina, J.L. 1999. Insulin inhibits growth hormone signaling via the growth hormone receptor/JAK2/STAT5B pathway. J. Biol. Chem. 274:13434-13442.

35. Menon, R.K., Arslanian, S., May, B., Cutfield, W.S. and Sperling, M.A. 1992. Diminished growth hormone-binding protein in children with insulindependent diabetes mellitus. J. Clin. Endocrinol. Metab. 74:934-938.

36. Mercado, M., and Baumann, G. 1995. Characteristics of the somatotropic axis in insulin dependent diabetes mellitus. Arch. Med. Res. 26:101-109.

37. Menon, R.K., et al. 1994. Tissue-specific regulation of the growth hormone receptor gene in streptozocin-induced diabetes in the rat. J. Endocrinol. 142:453-462.

38. Duran-Sandoval, D., Cariou, B., Fruchart, J.C., and Staels, B. 2005. Potential regulatory role of the farnesoid $\mathrm{X}$ receptor in the metabolic syndrome. Biochimie. 87:93-98.

39. Duran-Sandoval, D., et al. 2004. Glucose regulates the expression of the farnesoid $\mathrm{X}$ receptor in liver. Diabetes. 53:890-898.

40. Chartier, C., et al. 1996. Efficient generation of recombinant adenovirus vectors by homologous recombination in Escherichia coli. J. Virol. 70:4805-4810.

41. Fallaux, F.J., et al. 1996. Characterization of 911: a new helper cell line for the titration and propagation of early region 1 -deleted adenoviral vectors. Hum. Gene Ther. 7:215-222.

42. Conte, F., et al. 2002. Identification of a region critical for proteolysis of the human growth hormone receptor. Biochem. Biophys. Res. Commun. 290:851-857. 